\title{
Design of Greenhouse Temperature and Humidity Monitoring System Based on ZIGBEE Technique
}

\author{
Ming Xin $^{1, a}$, Wei Zhongshan ${ }^{1, b,{ }^{*}}$ \\ ${ }^{1}$ Department of Computer and Electronic Information Engineering, Guangxi Vocational and \\ Technical College , Nanning 530226, Guangxi, China \\ a 120722239@qq.com \\ b724704157@qq.com \\ ${ }^{*}$ Corresponding author
}

Keywords: ZigBee, CC2530, Greenhouse, Temperature and humidity collecting

\begin{abstract}
Based on ZIGBEE technique, this paper designs a monitoring system of environment parameters in greenhouse. ZigBee technique is firstly introduced, followed by the demonstration of overall design scheme of the monitoring system, mainly on the hardware and software composition of data acquisition and coordinator nodes whose core is CC2530, and on the work principle of temperature and humidity sensors and circuit design. Detailed analysis is made on the program design of every module. Test results show that all data nodes function well and can collect appropriate parameters with negligible errors; the wireless networking of all sensor and coordinator nodes works properly and can be used to transfer data, meeting the system design requirements. Therefore, it is proved that the system design is practical.
\end{abstract}

\section{Introduction}

Traditional monitoring system for agricultural greenhouses generally uses wire communication technology. But the intelligent measurement and control technology is still hard to be applied and promoted in real production, for the laying of communication wires and cables, the easily aging, unreliable, costly and hard maintaining cables.

ZigBee technology is an emerging wireless communication technology characterized by low price, low power consumption and automatically networking. It has been widely applied in mine, ports, food traceability, airports and other places. It is a short-distance wireless communication technology suitable to be applied in wireless sensor networks.

In view of the above, this paper designs a monitoring system based on ZigBee technology for greenhouse environment parameters.

\section{Overall design of the system}

The wall of this greenhouse is of plastic construction, with vents and fan in corners, and ceiling curtain and skylight on the top. The purpose of this paper is to use the ZigBee wireless network to design a wireless monitoring system for the greenhouse temperature and humidity, to achieve wireless intelligent monitoring of the temperature and humidity in the greenhouse.

In this system, data is firstly collected through temperature and humidity sensors. After data preprocessing, it is transferred to the ZigBee Coordinator via ZigBee ZigBee end-node through ZigBee network. Through the RS485 bus, ZigBee Coordinator sends the packaged data to the host computer. Monitoring and control software is run in the host computer to make real-time display of environment parameters collected from each node and store them for historical queries. Control system structure is shown in Figure 1: 


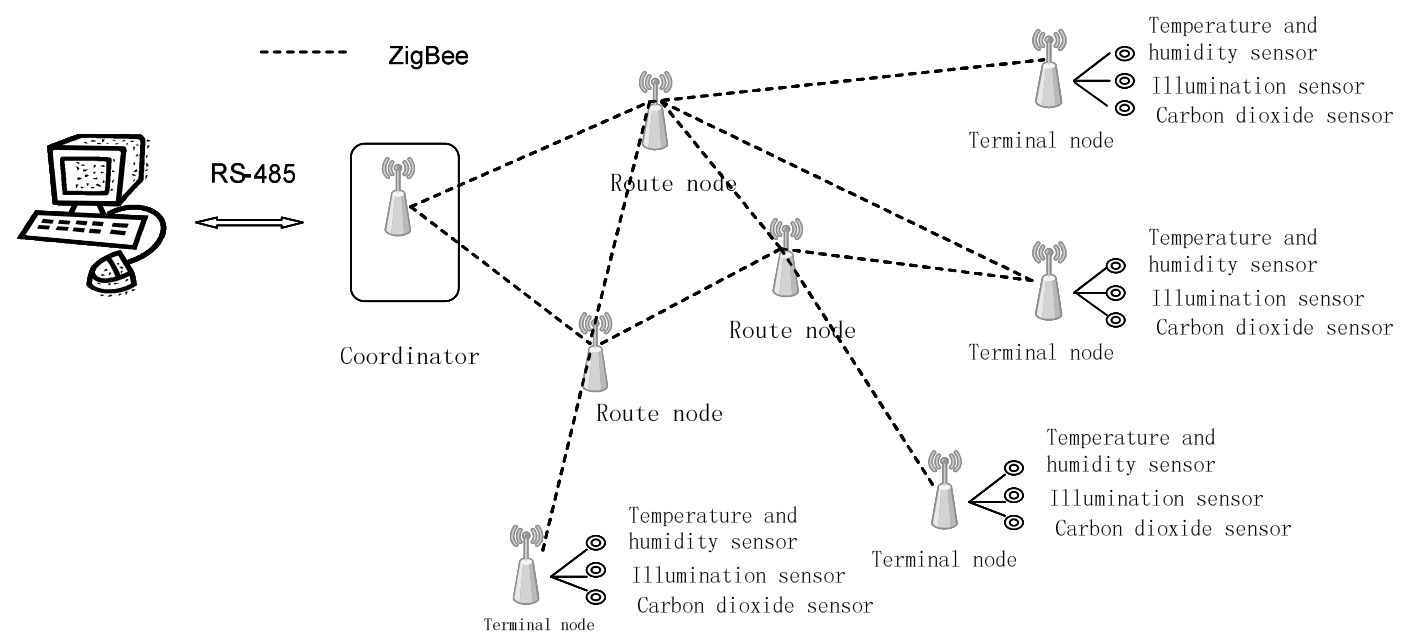

Fig.1. Structure chart of the control system

Coordinator nodes are the originators of the entire network, taking charge of managing the entire ZigBee networks. After receiving data collected by each sensor node, it sends the data to the host computer.

The main duty of data collection sub-nodes is to collect and preprocess the data of environment parameters, and then to send it to the coordinator.

\section{Hardware design of the system}

The hardware of this system is mainly made up from three parts: data collection circuit, wireless data transmission circuit and data communication interface circuit.

Design of data collection circuit. There are many different kinds of temperature sensors available in the market, such as thermocouple, RTD, integration temperature sensor and pyroelectric sensor. In this project, DHT11 is used as temperature and humidity sensors.

DHT11 digital temperature and humidity sensor is a composite temperature-humidity sensor which contains a calibrated digital output inside. It consists of a resistor humidity sensing element, a thermistor temperature element as well as an 8-bit single chip microcontroller. Therefore, DHT11 has good characteristics like free of external circuit, low energy consumption, long distance transmission, fast response, strong anti-interference and cost-effective. By applying a single-wire serial interface, the sensor simplifies its circuit. Moreover, it also has other properties like small size, low power consumption, long distance signal transmission. It has only 4 pins, which makes connection convenient.

The voltage supply of DHT11 is 3-5.5V. A 104 porcelain capacitor filter can be added between the power and ground. MCU is connected via data transmission pin used for communication between MCU and DHT11 using a single bus data format. The circuit diagram DHT11 is shown in Figure 2:

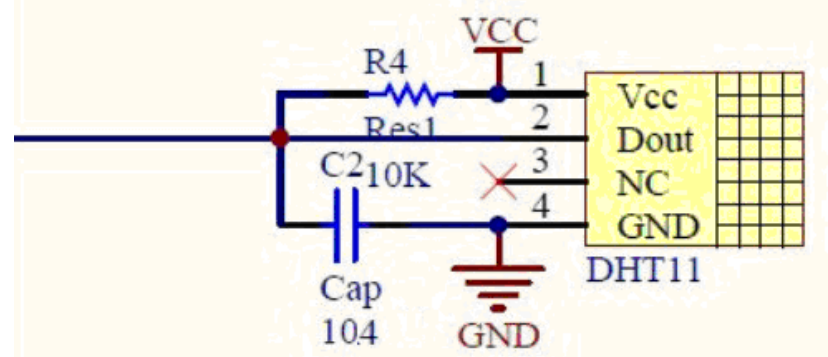

Fig. 2. Circuit diagram of DHT11 
Design of wireless data transmission circuit. Data transmission section is composed of multiple ZigBee modules. ZigBee module collects data acquired from all the nodes in a wireless way, and then transfers it to the computer for further processing and analysis.

In the process of system development, ZigBee chip selects SOC type. The CC2450 of TI uses 8051 micro kernel and it has been widely developed currently, reducing the difficulty of development. Therefore, considering a combination of factors, it is decided that ZigBee chip uses the TI CC2530 chip.

CC2530 is the second generation ZigBee 802.15.4 SOC chips introduced by Texas Instruments after the CC2430. It integrates industry-leading RF transceivers, enhanced industry standard 8051MCU in-system programmable Flash memory 8k Bytes of RAM and many other powerful features. CC2530 has four different versions of the Flash. The biggest one can provide $256 \mathrm{~K}$ program memory. The maximal transmitter power of CC2530 is up to $+4.5 \mathrm{dBm}$, its receiver sensitivity reaching $-97 \mathrm{dBm}$. And its working temperature ranges $-40 \sim+125$ degrees centigrade. The minimum system circuit of CC2530is shown in Figure 3:

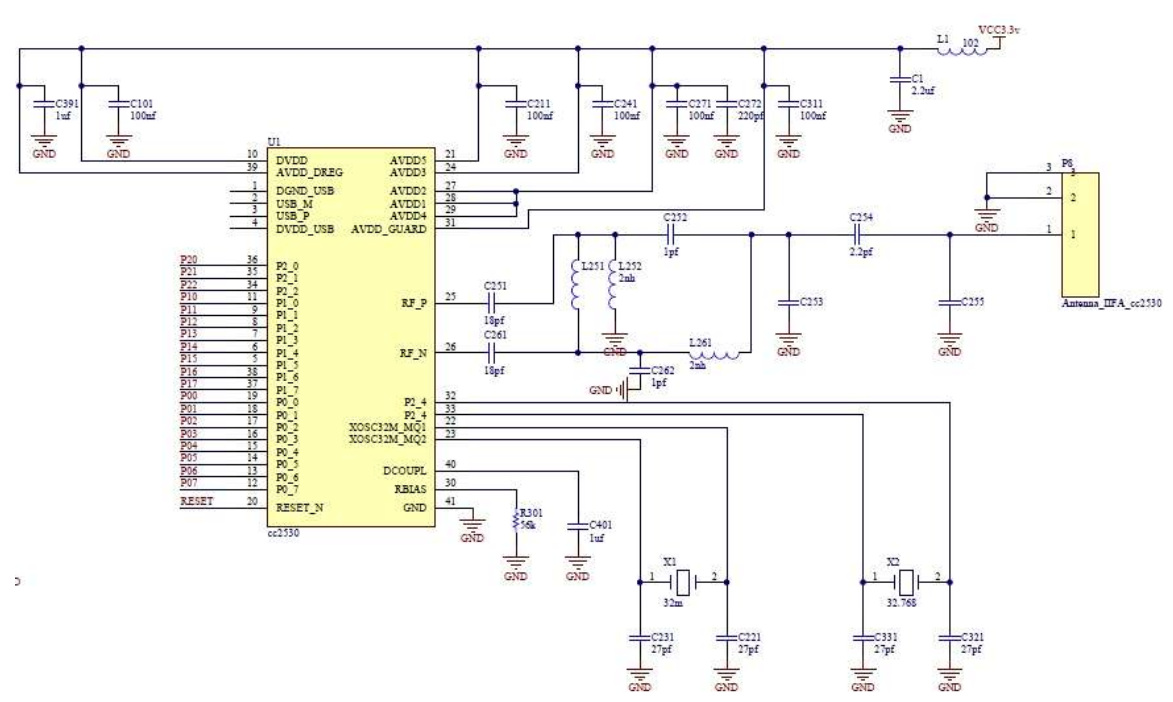

Fig.3. CC2530 circuit diagram

Design of data communication interface circuit. Coordinator node needs 485 bus to send data collected via Wi-Fi to the computer. Since CC2530 general IO port output high electrical level of 3.3 $\mathrm{V}$ and low level of $0 \mathrm{~V}$, a level conversion chip is needed to converse TTL level into 485-level. This system uses a MAX485 whose structure and pin are very simple. It contains a driver and receiver inside.

RO pin is the output end of the receiver, while the DI pins is the input end of the driver. When the MCU is in an asynchronous transmission mode, RXD and TXD pins are attached to the RO and $\mathrm{DI}$ respectively. DE and/RE pins are the receiving and sending ends of 485 . When $\mathrm{DE}$ is in high electrical level, the device is in transmission mode. When /RE is in low electrical level, the device is in receiving state. Since the sending and receiving of data on the RS485 bus is not simultaneous, MCU only needs one pin to control these two pins.

\section{Software design of the system}

Z-Stack program analysis. Z-Stack applies decentralized management framework. Physical layer and data link layer are at the bottom and are associated with hardware. In this design, they are decided by CC2530. Network layer, application support sub-layer and application layer and security layer operate above the physical layer and data link layer and are completely detached from the hardware. Such a structure makes program writing and debugging more convenient. 
Z-Stack is constructed from the perspective of OS. This operating system sticks to event round-robin rules. After every layer completes the initialization, OS enters low-power mode to wait for the event. When an event is triggered, system will wake up to extract messages in order to find the event-handling function entry and handle the event. Then it returns to low-power mode once again. If several events are triggered at the same time, they are dealt with according to the query order. The running process of Z-stack is as follows: electric starter on the system, the CC2530 initialization, OSAL initialization and startup, code entering an infinite loop and implementing tasks round-robin.

Design of coordinator node program. The core of ZigBee network is coordinator node, which take charge of establishing and managing the network. After electrifying the circuit board, the first step is to initialize the hardware and protocol suite. Next, coordinator continuously conducts channel-level scanning in order to select an appropriate channel to create a ZigBee network. When a new device applies for joining the network, the coordinator is in charge of allocating a short 16-bit address to it and allowing it to join the network. After the network is completed, the coordinator node receives upload of data from the data collection node and routers node, and then uploads the data to a host PC through the RS485 bus interface. The working process of coordinator node is shown in Figure 4:

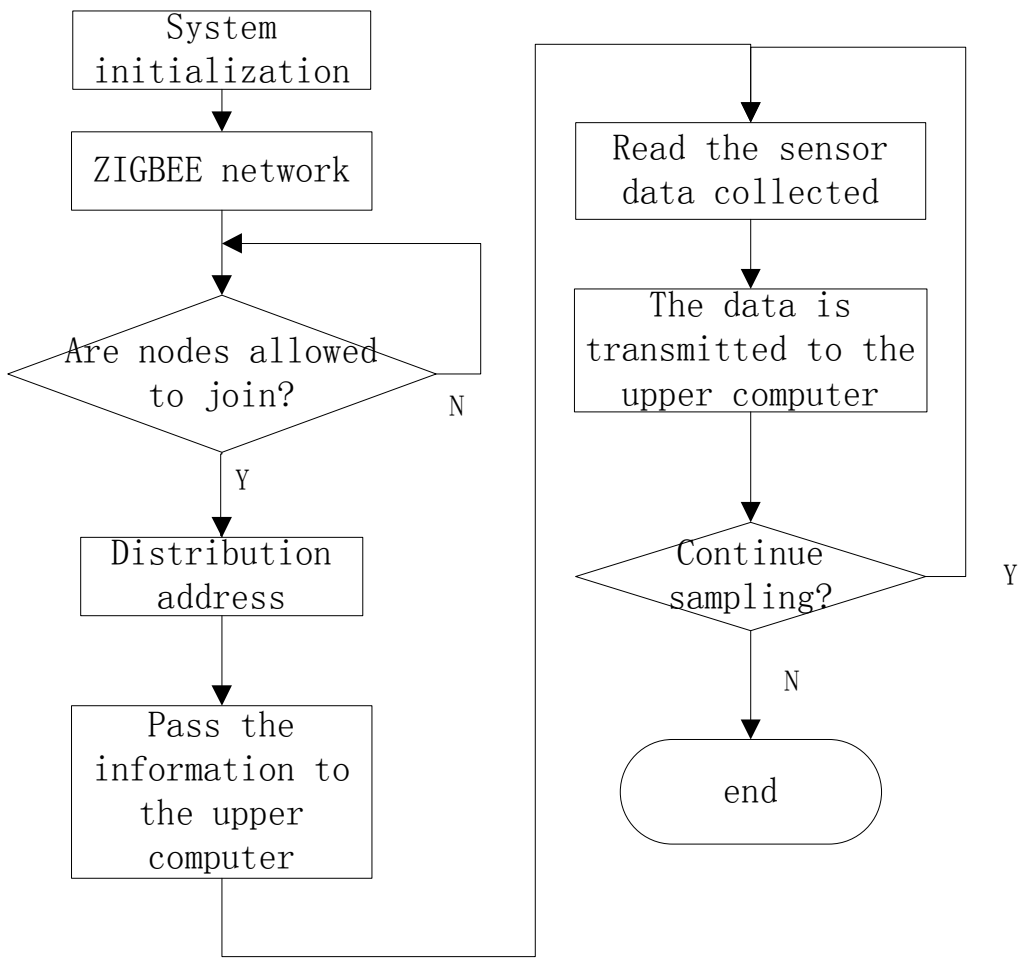

Fig. 4. program flow diagram of coordinator

The establishment of ZigBee network is initiated by the coordinator. A ZigBee device to create a ZigBee network must meet the following two requirements: it must be fully functional nodes (FFD), and must possess the capability of Zigbee Coordinator; it cannot join any other ZigBee network.

Programming of data collection node. The main function of data collection node is to transmit data collected from all the sensors to their parent nodes. After completing the initialization process of CC2530, protocol suites, and their respective connected sensors, data collection node starts scanning channels. When it finds proper network, it will apply for joining the network. After its application is confirmed, it shifts to sleep mode until the wake timer wakes it up. After the node is awake, it collects data from all the sensors and preprocesses those data, and then uploads it to the parent node. Data collection node program flow diagram is shown in Figure 5: 


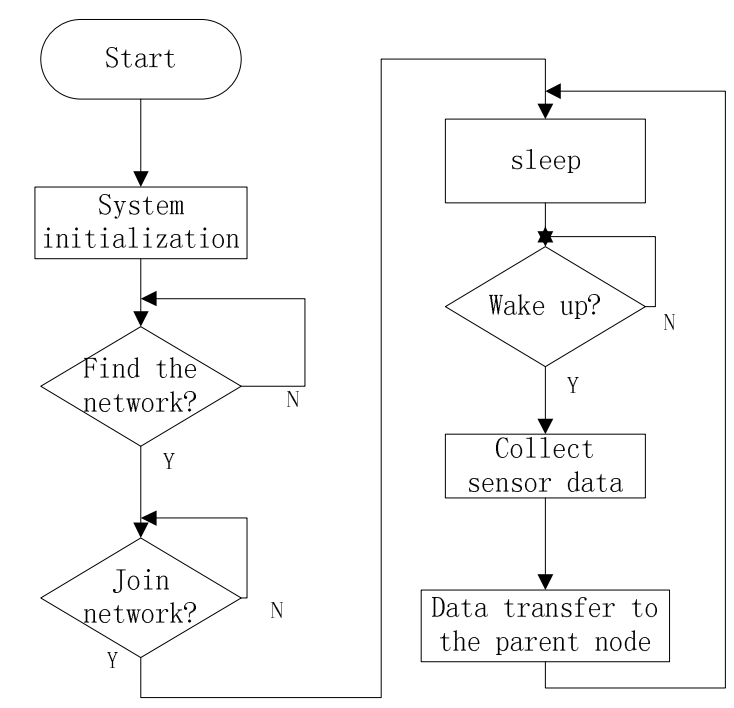

Fig. 5. Data collection node program flow diagram

Every Zigbee device has two network addresses: a 64bit address, also known as the MAC address or an IEEE address, is globally identification of Zigbee; and the other address is 16bit address, or logical address, also known as short address. On the establishment of a ZigBee network, the ZigBee coordinator will configure a logical address 16bit to each node in the network.

Electrified terminal nodes will automatically seek to apply for joining the Zigbee network, if a node is not expected to join the Zigbee network but to carry out other programs, the Hold_Auto_Start parameter in the configuration file should be modified. Users will also need to use the ZDAPP_STARTUpFromAPP () function to set how long before the device is allowed to join the Zigbee network.

\section{System testing}

Arrange Data collection point around the greenhouse; open the power supply of all modules; run host computer software to receive data transmitted by the Coordinator; draw temperature curve and save the data as an EXCEL file for query.

Once the system is run, the Coordinator keeps sending measured data to host computer. The host computer displays measurement data collect from all the nodes and draw corresponding parametric curve, and will save all the data into a spreadsheet in order to analyze historical data.

After the system runs properly, the host computer displays the connection status of all the nodes to show Coordinator nodes and data collection nodes are successfully connected and that data can be transferred properly.

In the process of testing, in order to verify the accuracy of sensor-collected data, thermometer and hygrometer is placed on node 1 in the greenhouse to artificially record temperature and humidity. Within two hours, 12 consecutive sets of data are recorded. The comparison of data gathered by the system (obtained from the software in historical data tables) and data artificially recorded is displayed in table 1.

\begin{tabular}{lllllllllllll} 
& \multicolumn{10}{c}{ Table 1 data analysis } \\
\hline $\begin{array}{l}\text { Collected } \\
\text { temperature }{ }^{\circ} \mathrm{C}\end{array}$ & 1 & 2 & 3 & 4 & 5 & 6 & 7 & 8 & 9 & 10 & 11 \\
$\begin{array}{l}\text { measured } \\
\text { temperature }{ }^{\circ} \mathrm{C}\end{array}$ & 18.2 & 18 & 18.5 & 19 & 18 & 19 & 19.5 & 20 & 20 & 18.5 & 19 & 19 \\
$\begin{array}{c}\text { Collected } \\
\text { humidity } \\
\quad(\mathrm{RH})\end{array}$ & 60 & 61 & 61 & 63 & 63 & 62 & 64 & 65 & 65 & 63 & 63 & 63 \\
$\begin{array}{c}\text { Measured } \\
\text { humidity } \\
(\mathrm{RH})\end{array}$ & 61 & 61 & 62 & 62 & 63 & 63 & 63 & 65 & 66 & 62 & 63 & 62 \\
\hline
\end{tabular}


According to the comparison, the temperature data error is less than $0.3^{\circ} \mathrm{c}$, and the humidity data error is less than $1 \%$, indicating that the gap between data collected by data collection node and the actual measurement is negligible.

Through on site testing, it is found that all sensor nodes and coordination devices can achieve Group networking and data transmission, and that data can be properly transmitted between coordination nodes and the host computer machine. The host computer can display parameters collected from all nodes and can save historical data electronic form, and can draw corresponding changes curve. Meantime, the comparison between artificial measured data and that collected from sensor nodes verifies the accuracy of collected data.

\section{Conclusion}

Based on ZIGBEE technique, this paper designs a monitoring system of environment parameters in greenhouse. Detailed analysis is made on overall design, the hardware and software design. On site testing is made to show that all data nodes function well and can collect appropriate parameters with negligible errors; the wireless networking of all sensor and coordinator nodes works properly and can be used to transfer data, meeting the system design requirements. Therefore, it is proved that the system design is practical.

Since this system is characterized by low price, low power consumption, easy to deploy and automatically networking, it can connect to different types of sensors to monitor warehouse, mine, airport, port and station. Therefore, it is of high practical value.

\section{References}

[1] Zhang Weibin, Research and Realization of Zigbee-based Remote Monitoring System for Greenhouse [D]. Northeast Petroleum University. (2014).

[2] Miao Lianqiang, Hu Huiping, Design of Remote Environment Monitoring System for Greenhouse Based on ZigBee Technology [J]. Instrumentation and sensors. 10 (2010).

[3] Ma Lin, Applications of Temperature Sensor [J]. The Electricity Era. 20 (2011).

[4] Chai Shilong, Liu Yi, Zhang Zhenhu. temperature and humidity monitoring system design based on ZigBee [j]. the Horizon 2015. (04)

[5] ChiPeonAS, SmartRFCC2530PreliminaryDatasheet. 2 (2009).

[6] Zhou Kai, Guo Lili. A Method to Realize Serial Communication Between SCM and PC Using MAX485 [J]. The Applied Science and Technology. 3 (2003).

[7] Information on http://www.zigbee.org,2009-10-15.

[8] Liu Tao, Ma Jixun, Lian Haitao. Using RS-485 Communication Protocol to Realsize Multipoint Communication Between PC and SCM [J]. Modern Electronic Technology. 5 (2002).

[9] Song Xiaoqin, Hu Aiqun. Network Data Link Layer and Network Layer Design in Wireless Sensor [J]. Telecommunications Science, 9 (2005) 9-12.

[10] Lan Xiao. Design and Implementation of Wireless Gas Meter Reading System Based on Zigbee Technology [D]. University of Electronic Science and Technology of China. (2007). 